3 Meisel J L, Bergman D, Graney D, Saunders D R, Rubin C E. Human rectal mucosa: proctoscopic and morphological changes caused by laxatives. Gastroenterology 1977; 72: 1274-9.

4 Bigard M A, Gaucher P, Lassalle C. Fatal colonic explosion during colonoscopic polypectomy. Gastroenterology 1979; 77: 1307-10.

Eric Hassall and Marvin Ament Division of Pediatric Gastroenterology, UCLA Center for the Health Sciences, 22-340 Marion Davies Children's Clinic, Los Angeles, California 90024, USA

\section{Dr Williams and Dr Walker-Smith comment :}

The practical comments offered by Hassall and Ament relating to paediatric colonoscopy are well taken. Any pathologist reviewing biopsies taken after bowel preparation should certainly expect to make allowance for a small increase in cellular infiltrate and significant mucus depletion. It is not our experience that either petechiae or significant erythema result from purgation or enemas, except for local trauma in the ano-rectal area.

The sweetness of mannitol makes it acceptable to children and there is no hazard if no electrosurgery is contemplated; some of the alternative saline/saline plus PEG solutions are unpalatable but other proprietary mixtures-such as Picolax-might be a satisfactory alternative. We too individualise the dose of pethidine and diazepam (Diazemuls is now preferable as it is painless and non-toxic) using the least dose of diazepam to obtain amnesia but a generous dose of pethidine titrated to the particular child's requirement (which is often impossible on a $\mathrm{mg} / \mathrm{kg}$ basis). We use naloxone for reasons of social convenience to the child and nursing staff for whom the very rapid reversal of sedation means that normal activities can be resumed within a few minutes of the end of the procedure.

The question as to who should perform colonoscopy in children is a more debatable one. There is no need for paranoia on this subject and doubtless the solution will be different in different centres according to the resources and staff available. It is our policy that paediatric gastroenterologists should be trained to do as much as possible and that most endoscopic procedures should certainly be done in the surroundings of the paediatric unit with which the child is already familiar. In many of our cases however, there is a positive indication for total colonoscopy including taking of biopsies in the terminal ileum. Limited examination is sometimes clinically sufficient and can easily be performed by the paediatrician, often without any sedation. Some total colonoscopies are surprisingly difficult and when a major centre such as UCLA Division of Paediatric Gastroenterology performs only an average of 13 colonoscopies a year it is difficult to imagine that many other paediatricians will acquire the dexterity or 'tricks of the trade' acquired by a specialised adult gastroenterologist performing at least a thousand procedures a year. It may be as a result of this facility that we perform about a hundred paediatric total colonoscopies a year as well as numerous limited examinations performed in the ward or outpatient clinic by paediatricians alone.
Although snare polypectomy and the use of the electrosurgical unit may present no problem to the paediatric surgeon it is doubtful if many paediatric gastroenterologists can become sufficiently practised with the apparatus and its use to be really safe. We agree fully with the need to stimulate the growth of paediatric fibre-endoscopy in general and colonoscopy in particular, but it would be a pity if a spirit of chauvinism meant that paediatric gastroenterologists should wish to develop their endoscopic skills in isolation without the benefit of all the many lessons that their adult colleagues have learnt and would willingly pass on.

We take it that the comment that the UCLA colonoscopies and polypectomies 'have been performed by our paediatric gastroenterology nursing staff' is a misprint.

\section{Which infants should not receive intensive care?}

Sir,

I wish to point out a grave anomaly in an otherwise admirable article. ${ }^{1}$ Campbell is right in saying that decisions to withhold or withdraw intensive care are 'complex because they concern judgments that not only are medical but are moral, ethical, and legal and for which doctors have little or no formal training' (page 569). The article itself undeniably shows that doctors, even complete neonatal teams (all of whom I presume have little or no formal training) have, nevertheless, learned from experience most moral wisdom there is to be gained. Most of it or all of it? Campbell seems to claim the latter. He finds it "difficult to see how a "child advocate", an ethics committee, or the Courts could do any better"' (page 571). I wish to take exception to this statement as regards the ethics committees. Here, as elsewhere, the proof will be in the eating of the pudding.

Current ethics commitees often have an audit function only, that is they review problems of the past. Why would they not be 'on call' to play an active role in treatment decisions? Thus they would meet a need so well described by some Canadian paediatricians who feel that regional, national, or local groups could advise concerning ethical decisions, in particular clinical situations. What would be the contribution of such groups to the decision and its implementation?

Firstly, an ethics committee would function at some distance from the immediate crisis centre. Being less directly involved will raise the committee's chances for objective and independent evaluation.

Secondly, over and above the consistency with the patient's or the team's conviction-which seems to be the main moral concern of decision making according to Campbell-the committee should be able to survey the total picture. Thus, the ethics committee, besides confirming the medical team's approach to individual and micro-ethical questions, would give due consideration to the macro-ethical aspects of immediate, short- or longterm range. It is doubtful that the average neonatology team has the expertise for such exploration.

Thirdly, it is likely that a decision to which an ethics committee made its contribution, would be more readily accepted because of its moral competency and authority. 
Fourthly, the tragic situations under debate are, fortunately, rare. Yet they cause tremendous turmoil and moral agony. The neonatologist who told me 'we want to be sure that we did the right thing' expressed a need for moral confirmation of a medical decision. Debriefing of entire teams after the event may be helpful. However, reinforcement of the decision before its implementation could prevent the need for such debriefing.

Campbell is right when he says that 'to apply modern treatments indiscriminately ... . would be irresponsible' (page 571). Yet, since discernment displayed in current decisions has come more than once under heavy fire, the reasonable thing seems to be to mobilise those moral forces before deciding, rather than facing them after the facts.

\section{Reference \\ 1 Campbell A G M. Which infants should not receive intensive care? Arch Dis Child 1982; 57: 569-71.}

Maurice A M De WachTer Clinical Research Institute of Montreal, Centre for Bioethics, 110 Avenue des Pins Ouest, Montreal, Quebec H2W 1R7, Canada

\section{Effects of oral theophylline and oral salbutamol in the treatment of asthma}

Sir,

The negative interaction between salbutamol and theophylline shown in the study by Dawson and Fergusson $^{1}$ does not accord with my clinical experience ${ }^{2}$ s and can be explained by either of the following: (1) The use of an unsuitable oral formulation for the salbutamoltheophylline combination diminished the bio-availability of theophylline, consequently producing lower serum levels and thus less improvement in peak flow. (2) The response to a bronchodilator is dependent on the baseline peak flow value, the poorest response being obtained in both the most mild and most severe forms of asthma. The best way to gauge this response is to measure the percentage improvement over the baseline value. In their study Dawson and Fergusson neither measured baseline values nor did they record the percentage improvement. Although assignment was random the severity of the asthma (mean baseline values) between the two groups could have been significantly different.

For the sake of the record it should be pointed out that the maximum recommended dose of salbutamol is $0 \cdot 15$ and not $0 \cdot 1 \mathrm{mg} / \mathrm{kg}{ }^{4}$

\section{References}

1 Dawson K P, Fergusson D M. Effects of oral theophylline and oral salbutamol in the treatment of asthma. Arch Dis Child 1982; 57: 674-6.

2 Blumenthal I, Berry D. Dosage guidelines for slow-release aminophylline in childhood asthma. Br J Clin Pharmacol 1980; 9: 526-7.

3 Blumenthal I. A comparative trial of slow-release aminophylline, salbutamol, and a half dose combination in the prevention of childhood asthma. J Int Med Res 1980; 8: 400-3.
4 Connolly N M. Dosage of oral salbutamol in asthmatic children. Arch Dis Child 1971; 46: 869-71.

IVAN BLUMENTHAL Oldham and District General Hospital, Rochdale Road, Oldham OLI $2 \mathrm{JH}$ 产

Dr Dawson and Dr Fergusson comment:

We regret that the results of our randomised double of blind trial do not accord with Blumenthal's experience $\vec{O}$ and, of course, we would welcome a replication of our results. In reply to his specific comments: (1) Whether or $\vec{\omega}$ not the oral formulations of theophylline and salbutamol $\stackrel{\rho}{\sim}$ used were unsuitable and diminished the bio-availability of theophylline is a matter for further investigation. However, in our trial we used standard oral preparations $\mathcal{O}_{\infty}$ of Ventolin syrup and Nuelin syrup which are commonly $\rightarrow$ used in the treatment of asthma, and we have little doubt that many patients on combination therapy receive these specific preparations. Our results are thus likely to reflect the effects of typical prescribing patterns. (2) Since 5 the major aim of our trial was to examine the hypothesis of Wilson et al. ${ }^{1}$ that when used in combination salbutamol and theophylline produced increased serum $\vec{r}$ theophylline levels, measures of peak flow were collected only incidentally during the course of the trial. Therefore, we cannot comment on whether similar effects would be $\vec{\oplus}$ shown with a percentage improvement statistic. However, Blumenthal's comments appear to have missed the major point of our analysis which was not to demonstrate differences in single measures but rather differences in joint, multivariate, distribution of serum levels, peak flow rates, and pulse rates between treatment groups. The compelling aspect of the study is the way in which all $\mathbb{D}$ three indices behave in similar ways between the two treatments rather than the results for single measures.

Dr Blumenthal's comments 'Although assignment was 3 random ... the two groups could have been significantly different' betray a misunderstanding of the logical basis of the test of significance applied to a randomised experiment. Statements such as ' $\mathbf{P}<0.05$ ' simply imply that $\stackrel{\circ}{\circ}$ the probability of the observed results is less than $5 \% \div$ under the null hypothesis that there were no differences 3 between the treatment groups. Implicit in this logic is the

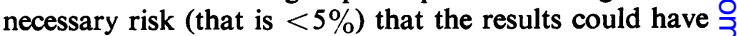
been due to the way subjects were assigned to treatments. This risk is taken once the null hypothesis is rejected and $\mathrm{O}$ is part and parcel of the decision theory associated with $D$ the significance test. We would add, however, that with respect to pre-trial serum theophylline levels there were no significant differences between the groups. The mean $O$ pre-trial level for the theophylline group was $8.46 \mathrm{~N}$ $\mu \mathrm{mol} / \mathrm{l}$, compared with the mean of $7 \cdot 4 \mu \mathrm{mol} / \mathrm{l}$ for the salbutamol/theophylline groups.

We thank Dr Blumenthal for correcting our impressions of the maximum recommended dosages for salbutamol, $\stackrel{\circ}{\varnothing}$ although in recent trials it has been customary to use a $\underset{\varnothing}{\overparen{D}}$ $0.1 \mathrm{mg} / \mathrm{kg}$ dosage. ${ }^{2}$

\section{References}

1 Wilson J D, Sutherland D C, Thomas A C. Has the change to beta-agonists combined with oral theophylline $\stackrel{\rho}{T}$ increased cases of fatal asthma? Lancet 1981 ; i: 1235-7. 